\title{
Tissue mimicking phantoms for fluorescence imaging
}

Shupletsov, Valery, Kandurova, Ksenia, Stavtsev, Dmitry, Stolbov, Aleksandr, Potapova, Elena, et al.

Valery Shupletsov, Ksenia Kandurova, Dmitry Stavtsev, Aleksandr Stolbov, Elena Potapova, Viktor Dremin, Andrey Vinokurov, Konstantin Podmaster'ev, Andrey Dunaev, "Tissue mimicking phantoms for fluorescence imaging," Proc. SPIE 11457, Saratov Fall Meeting 2019: Optical and Nano-Technologies for Biology and Medicine, 1145708 (9 April 2020); doi: 10.1117/12.2564389 


\title{
Tissue mimicking phantoms for fluorescence imaging
}

\author{
Valery Shupletsov ${ }^{a^{*},}$ Ksenia Kandurova ${ }^{a}$, Dmitry Stavtsev ${ }^{a}$, Aleksandr Stolbov ${ }^{a}$, Elena Potapova ${ }^{\mathrm{a}}$, \\ Viktor Dremin $^{\mathrm{a}, \mathrm{d}}$, Andrey Vinokurov ${ }^{\mathrm{c}}$, Konstantin Podmaster'ev ${ }^{\mathrm{b}}$, Andrey Dunaev $^{\mathrm{a}}$ \\ ${ }^{a}$ Research and Development Center of Biomedical Photonics, Orel State University, Russia; \\ ${ }^{\mathrm{b}}$ Orel State University, Russia; \\ 'Department of Industrial Chemistry and Biotechnology, Orel State University, Russia; \\ ${ }^{\mathrm{d}}$ Optoelectronics and Measurement Techniques Unit, University of Oulu, Finland;
}

\begin{abstract}
The article describes the development of the optical phantom mimicking flavin adenine dinucleotide (FAD) and skin collagen fluorescence. The results of experimental studies using fluorescence imaging and fluorescence spectroscopy methods are demonstrated. The method of optical phantom production for quick calibration of fluorescence imaging devices for further application in clinical practice is proposed.
\end{abstract}

Keywords: optical phantom, optical biopsy, fluorescence imaging, fluorescence spectroscopy, riboflavin, gelatin.

\section{INTRODUCTION}

Oncological diseases rank second in mortality worldwide ${ }^{1}$. According to the World Health Organization statistics, almost 18 million new cases of cancer were detected in 2018 and 9.6 million deaths from cancer occurred. One of the causes of this situation is difficulties of cancer diagnosis in the course of the treatment. Detection and differentiation of tumors before and during surgical treatment is usually done by visual inspection followed by a biopsy of suspicious tissue sites. Histological examination of the samples taken takes quite a long time, however, during the operation itself, it is difficult to detect all areas of tumor infiltration.

Modern preclinical and clinical studies show that application of optical imaging methods may allow the surgeon to receive more detailed information on the localization of tumor tissue in real time, which can significantly improve the quality of surgical interventions by reducing a false negative results rate. Methods of fluorescence spectroscopy (FS) and fluorescence imaging (FI) demonstrate significant sensitivity to the presence of pathological changes in tissues, in particular malignant ones ${ }^{2-5}$. While the FS and FI approaches can be successfully used for the fluorescence contrasting at selected wavelength of the emission ${ }^{6,7}$, the transcutaneous fluorescence measurements of endogenous fluorophores in living tissues can be challenging due to the intersection of the emission spectra and variable absorption. Extensive studies have been conducted in this direction to develop methodologies and devices for effective distinguishing of normal and tumor tissues in various human organs (lungs, breast, colon, cervix, etc.) ${ }^{8-11}$.

It is known that cells undergo certain transformations during carcinogenesis leading to morphological and biochemical changes, which can be detected by fluorescence diagnostic methods. Active proliferation under hypoxia conditions forces tumor cells to switch to the glycolytic type of metabolism. A characteristic feature of tumor cells in this case is an increase in the concentration of nicotin adenine dinucleotide (NADH), change in redox ratio of NADH and flavin adenine dinucleotide (FAD), as well as the accumulation of porphyrins ${ }^{12-14}$. Several clinical studies of FAD showed that changes in its fluorescence are associated with pathological processes in the tissues ${ }^{15}{ }^{16}$. As FAD is one of cofactors participating in various processes of cellular metabolic activity (tricarboxylic acid cycle, oxidative phosphorylation, acetyl coenzyme A synthesis, etc. $)^{17}$, the increase in FAD fluorescence can be explained by an increase in metabolic activity in abnormal tissues ${ }^{18}$, which, compared with healthy biological tissue, indicates tumor growth ${ }^{19}$.

However, biological tissues are heterogeneous complex structures containing various fluorophores in different concentrations and at different depths ${ }^{20,21}$. To correctly interpret the experimental data and determine the contribution of each fluorophore in total signal, it is necessary to create optical phantoms. Optical phantoms possess optical properties close to biological tissues to simulate typical interaction of optical radiation with biological tissue, in case of our work,

* matoka_97@mail.ru; phone +7 953 6111625; http://www.bmecenter.ru/en 
the fluorescence process ${ }^{22}$. Therefore, the aim of this work was to create an optical phantom mimicking the fluorescence of biological tissue, which can be used to calibrate a FI system.

\section{MATERIALS AND METHODS}

Optical phantoms described in most publication are based either on a liquid structure, which allows one to model infinite or semi-infinite single-layer media with absorption, scattering, and fluorescence effects ${ }^{23}, 24$, or on a solid base, which allows one to preserve the desired geometric shape, thickness, and heterogeneity, such as in the case of multilayer fabrics ${ }^{25,26}$ The optical phantom created in this work includes both a solid base for collagen fluorescence modeling and FAD fluorescence modeling fluid.

To produce optical phantoms simulating collagen fluorescence, it is possible to use gelatin - a hydrolyzed form of collagen. In the presented work, a multilayer model was created, consisting of $<1 \mathrm{~mm}$ thick sheets of edible gelatin of $<5$ $\mathrm{g}$ weight. The optimal number of gelatin sheets was experimentally selected by comparing the fluorescence intensity levels of gelatin sheets and skin collagen.

To simulate the FAD fluorescence spectrum, a $1 \%$ solution $(26.57 \mu \mathrm{M} / \mathrm{g})$ of riboflavin mononucleotide was used. This substance belongs to flavin group and has the fluorescence spectrum similar to the spectrum of FAD. Riboflavin has a higher quantum yield, and, unlike FAD, its quantum yield within $\mathrm{pH}$ 4-8 does not depend on acidity ${ }^{27}$. It is known that the concentration of FAD in human body usually varies from 0.1 to $1 \mu \mathrm{M} / \mathrm{g}^{28}$. In accordance with this data, four concentrations of riboflavin were selected close to the actual concentrations of FAD. The solution of riboflavin mononucleotide was diluted with distilled water in the following ratios: 1:32, 1:64, 1: 128 and 1: 256. Thus, the optical phantom was composed of two sheets of gelatin and the solutions of four different concentrations $(0.84 ; 0.42 ; 0.21 ; 0.1$ $\mu \mathrm{M} / \mathrm{g}$ ) of riboflavin were distributed in four different areas of the phantom.

The developed phantom was studied using an experimental setup implementing FS and FI methods (Fig. 1).

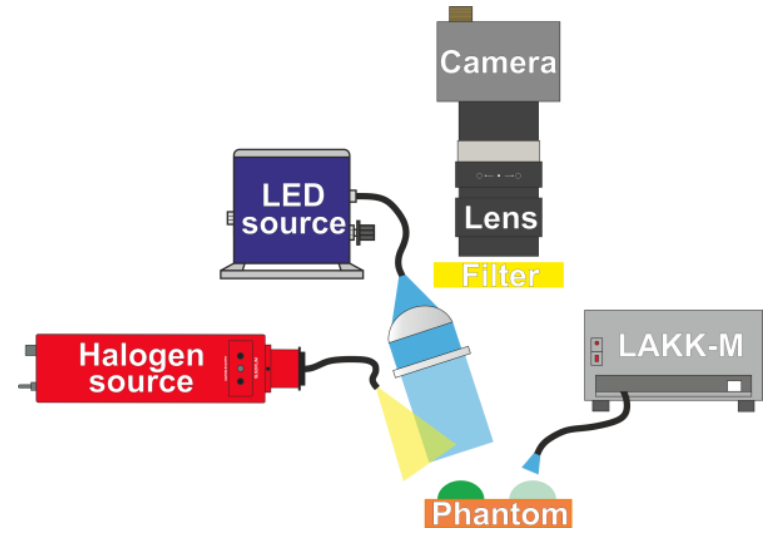

Figure 1. The general scheme of the experimental setup.

Radiation from a $455 \mathrm{~nm}$ LED source LLS-455 (Ocean Optics, USA) passes through a condenser and MF445-45 bandpass filter (Thorlabs, Inc. USA) and directed to an optical phantom to excite the fluorescence of gelatin and riboflavin. In FI channel, backscattered radiation of the source is removed by the FGL495 cut off filter (Thorlabs, Inc. USA) and the remaining fluorescence radiation is detected using a 340M-USB monochrome CCD camera (Thorlabs, Inc. USA) with a high frame rate and a 16 bit resolution. In FS channel, riboflavin fluorescence spectra are recorded using an optical fiber connected to the spectrometer of the LAKK-M multifunctional laser diagnostic complex (SPE "LAZMA" Ltd, Moscow, Russia). A stabilized broadband radiation source of 360-2600 nm range SLS201L-M (Thorlabs, Inc. USA) is used to obtain white light images. The methodology and process of experimental measurements are shown schematically in Figure 2. 


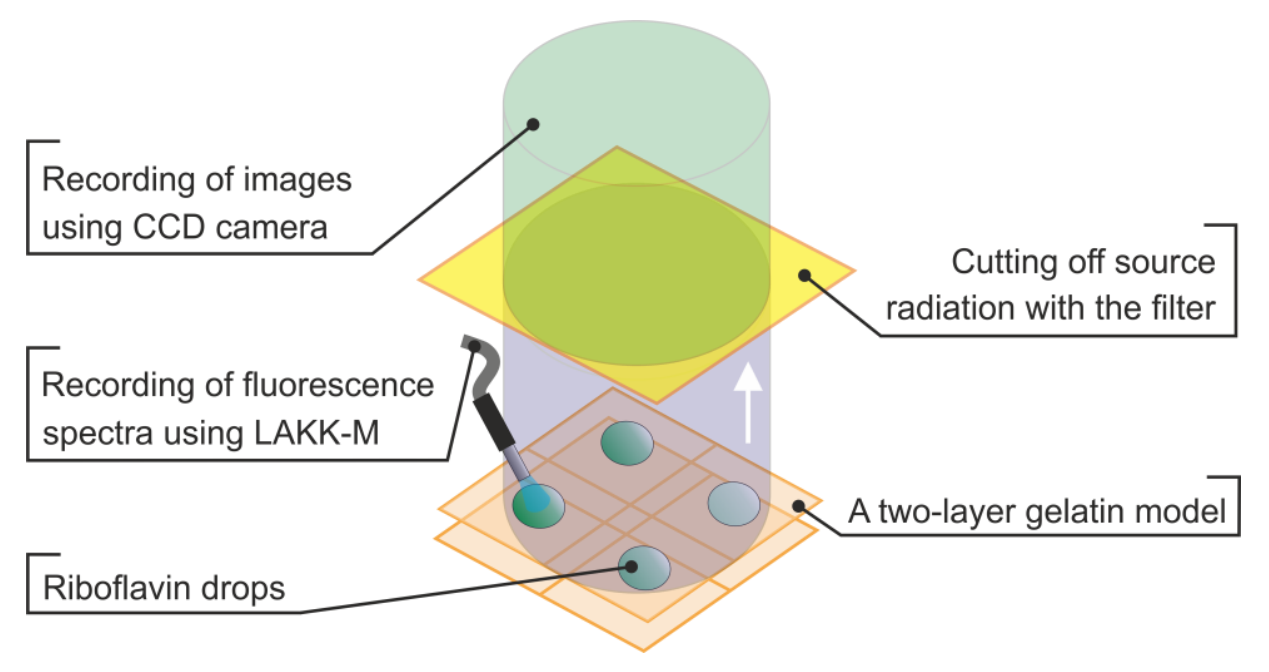

Figure 2. Research methodology.

The research methodology includes the sequential registration of fluorescence using the CCD camera and the spectrometer. Drops of the same volume $(20 \mu \mathrm{l})$ with different concentrations of riboflavin were applied to gelatin sheets using a micropipette. The optical phantom was located on a non-fluorescent non-reflective surface. At the first stage, a $495 \mathrm{~nm}$ filter was installed in front of the camera and fluorescence images were recorded 3 times. Then the image of the phantom in white light was recorded without a filter. At the second stage, the fluorescence spectra of each drop of riboflavin were recorded 3 times. The results were processed on a personal computer using specially developed software in Matlab environment.

\section{RESULTS AND DISCUSSION}

As a result, the image of an optical phantom in white light was obtained (Fig. 3, a), as well as fluorescence images under illumination by an LED source (Fig. 3, b) and images contrasted in pseudocolor (Fig. 3, c) with an intensity scale from 0 to 16000 . The image in white light was recorded with exposition time of 0.02 seconds, fluorescence images were recorded with 0.4 seconds exposition time.
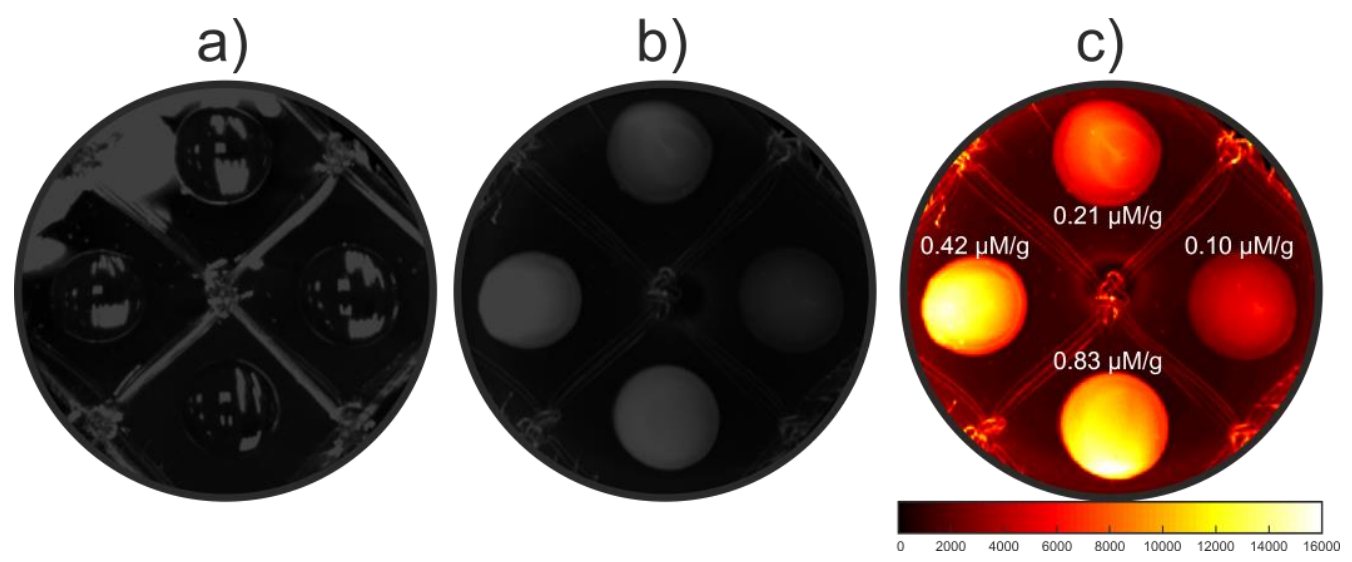

Figure 3. Images of an optical phantom: (a) image in white light, (b) fluorescent image, (c) contrasted fluorescent image.

In addition, the intensity values of riboflavin fluorescence were averaged in similar areas of each drop. The data on the maximum fluorescence intensity values obtained with the spectrometer were averaged as well and compared with the 
results of FI. The results of measurements and calculations of fluorescence intensity for four concentrations of riboflavin using both optical modalities are presented in Figure 4.

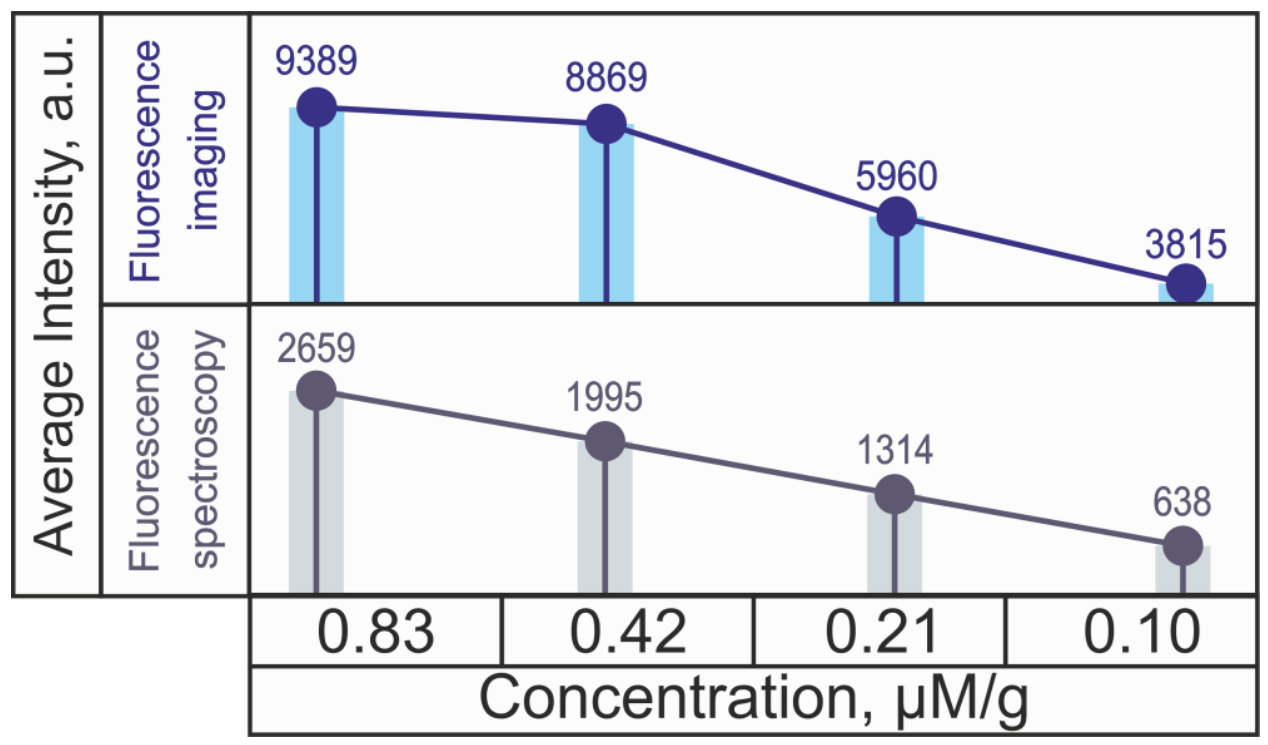

Figure 4. Average values of fluorescence intensity measured using FI and FS methods.

For the three lowest concentrations of riboflavin solutions, it was noticed that the fluorescence intensity recorded by FI decreases in proportion to the decrease in riboflavin concentration. As for the fluorescence intensity of the droplet with the highest concentration, the disproportionality of the measurement result indicates the need for exposure time correction during further measurements, which clearly shows one of the future functions of the phantom developed in this work. Fluorescence imaging data were also confirmed during measurements with a fluorescence spectroscopy channel. Future studies will be dedicated to the combining of the hyperspectral measurements ${ }^{29}$ with the fluorescence measurements to effectively compensate the influence of the presence of the tissue/phantom absorbers on the registering fluorescence intensity.

\section{CONCLUSION}

As the result of this work, it was showed that the proposed phantom is relatively easy to produce and use for the measurements. The phantom simulates the tissue fluorescence for specific research tasks, taking into account the effect of collagen presence. As an option for the further research on this direction, it is planned to develop a more complete optical model of biological tissue using other endogenous fluorophores, such as NADH and FAD themselves, porphyrins, etc.

The obtained experimental data indicate the sensitivity of the developed fluorescence imaging unit to the changes in the concentration of flavins. The use of the developed optical phantom will allow us to validate fluorescence imaging systems more accurately to improve the quality of implementation of this technology. The data obtained will be used to correct fluorescence images of pathological biological tissues and to more clearly determine the boundaries of a developing tumor.

\section{ACKNOWLEDGEMENTS}

The work has been funded by the Russian Foundation for Basic Research according to the research project 18-02-00669. 


\section{REFERENCES}

[1] Ferlay J., Soerjomataram I., Ervik M., Dikshit R., Eser S., Mathers C., Rebelo M., Parkin D. M., Forman D., Bray F., "GLOBOCAN 2012 v1. 0, cancer incidence and mortality worldwide: IARC CancerBase No. 11," Lyon, Fr. Int. agency Res. cancer 2016 (2013).

[2] E. Borisova, A. Gisbrecht, T. Genova-Hristova., "Multispectral autoflourescence detection of skin neoplasia using steady-state techniques" Proc. SPIE 1104704, (2019).

[3] Y.A. Khristoforova, I.A. Bratchenko, O.O. Myakinin., "Portable spectroscopic system for in vivo skin neoplasms diagnostics by Raman and autofluorescence analysis," J. of Biophotonics 12(4), e201800400 (2019).

[4] Dunaev A. V., Dremin V. V., Zherebtsov E. A., Rafailov I. E., Litvinova K. S., Palmer S. G., Rafailov E. U. "Individual variability analysis of fluorescence parameters measured in skin with different levels of nutritive blood flow," Medical engineering \& physics 37(6), 574-583 (2015).

[5] Kandurova K., Dremin V., Zherebtsov E., Potapova E., Alyanov A., Mamoshin A, Dunaev A "Fiber-optic system for intraoperative study of abdominal organs during minimally invasive surgical interventions," Applied Sciences 9(2), 217 (2019).

[6] Stelmashchuk O., Zherebtsov E., Zherebtsova A., Kuznetsova E., Vinokurov A., Dunaev A., "Noninvasive control of the transport function of fluorescent coloured liposomal nanoparticles" Laser Phys. Lett. 14(6), (2017).

[7] Tarakanchikova Y., Stelmashchuk O., Seryogina E., Piavchenko G., Zherebtsov E., Dunaev A., Meglinski I. "Allocation of rhodamine-loaded nanocapsules from blood circulatory system to adjacent tissues assessed in vivo by fluorescence spectroscopy" Laser Phys. Lett. 15(10), 105601 (2018)

[8] Alfano R., Tang G. C., Pradhan A., Lam W., Choy D., Opher E., "Fluorescence spectra from cancerous and normal human breast and lung tissues" IEEE Journal of Quantum Electronics 23(10), 1806-1811 (1987).

[9] Kolli V., Shaha A. R., Savage H. E., Sacks P. G., Casale M. A., Schantz S. P., "Native cellular fluorescence can identify changes in epithelial thickness in-vivo in the upper aerodigestive tract" The American journal of surgery 170(5), 495-498 (1995).

[10] Ramanujam N., Mitchell M., Mahadevan A., Thomsen S., Silva E., Richards-Kortum R., "Fluorescence spectroscopy: a diagnostic tool for cervical intraepithelial neoplasia (CIN)" Gynecologic Oncology 52(1), 31-38 (1994).

[11] Römer T. J., Fitzmaurice M., Cothren R. M., Richards-Kortum R., Petras R., Sivak M. V., "Laser-induced fluorescence microscopy of normal colon and dysplasia in colonic adenomas: implications for spectroscopic diagnosis" American Journal of Gastroenterology 90(1), (1995).

[12] Currie E., Schulze A., Zechner R., Walther T.C., Farese R.V., "Cellular fatty acid metabolism and cancer" Cell Metabolism 18(2), 153-161 (2013).

[13] Fox C.J., Hammerman P.S., Thompson C.B., "Fuel feeds function: energy metabolism and the T-cell response" Nature Reviews Immunology 5(11), 844-852 (2005).

[14] Moesta K.T., Ebert B., Handke T., Nolte D., Nowak C., Haensch W.E., Pandey R.K., Dougherty T.J., Rinneberg H., Schlag P.M., "Protoporphyrin IX occurs naturally in colorectal cancers and their metastases" Cancer research 61(3), 991-999 (2001).

[15] Wu Y., Qu J. Y., "Autofluorescence spectroscopy of epithelial tissues" Journal of biomedical optics 11(5), 054023 (2006).

[16] Wu Y., Xi P., Qu J., Cheung T., Yu M., "Depth-resolved fluorescence spectroscopy of normal and dysplastic cervical tissue" Optics express 13(2), 382-388 (2005).

[17] Lukina M., Shirmanova M., Sergeeva T., Zagaynova E., "Metabolic imaging in the study of oncological processes" Modern technologies in medicine 8(4), (eng) (2016).

[18] Chang S., Marín N., Follen M., Richards-Kortum R., "Model-based analysis of clinical fluorescence spectroscopy for in vivo detection of cervical intraepithelial dysplasia" Journal of biomedical optics 11(2), 024008 (2006).

[19] Pavlova I., Sokolov K., Drezek R., Malpica A., Follen M., Richards-Kortum R., "Microanatomical and Biochemical Origins of Normal and Precancerous Cervical Autofluorescence Using Laser-scanning Fluorescence Confocal Microscopy" Photochemistry and photobiology 77(5), 550-555 (2003).

[20] Meglinski I. V., Matcher S. J., "Quantitative assessment of skin layers absorption and skin reflectance spectra simulation in the visible and near-infrared spectral regions" Physiological measurement 23(4), 741 (2002). 
[21] Thueler P., Charvet I., Bevilacqua F., Saint Ghislain M., Ory G., Marquet P., Depeursinge C., "In vivo endoscopic tissue diagnostics based on spectroscopic absorption, scattering, and phase function properties" Journal of biomedical optics 8(3), 495-504 (2003).

[22] Potapova E., Dremin V., Zherebtsov E., Podmasteryev K.V., Dunaev A., "Liquid optical phantom for fluorescence spectroscopy," Fundamental and Applied Problems of Technics and Technology 331(5), 105-114 (2018).

[23] Beck G., Akgün N., Rück A., Steiner R., "Design and characterisation of a tissue phantom system for optical diagnostics" Lasers in medical science 13(3), 160-171 (1998).

[24] Wolf M., Keel M., Dietz V., Siebenthal K., Bucher H., Baenziger O., "The influence of a clear layer on nearinfrared spectrophotometry measurements using a liquid neonatal head phantom" Physics in Medicine \& Biology 44(7), 1743 (1999).

[25] Hielscher A., Liu H., Chance B., Tittel F. K., Jacques S., "Time-resolved photon emission from layered turbid media" Applied optics 35(4), 719-728 (1996).

[26] Kienle A., Glanzmann T., Wagnieres G., Bergh H., "Investigation of two-layered turbid media with timeresolved reflectance" Applied optics 37(28), 6852-6862 (1998).

[27] Drössler P., Holzer W., Penzkofer A., Hegemann P., "pH dependence of the absorption and emission behaviour of riboflavin in aqueous solution" Chemical Physics 282(3), 429-439 (2002).

[28] Aliverti A., Curti B., Vanoni M. A., "Identifying and quantitating FAD and FMN in simple and in iron-sulfurcontaining flavoproteins" Flavoprotein protocols - Humana Press 9 23, (1999).

[29] Zherebtsov E., Dremin V., Popov A., Doronin A., Kurakina D., Kirillin M., Bykov A., "Hyperspectral imaging of human skin aided by artificial neural networks" Biomed. Opt. Express 10(7), 3545 (2019) 